\title{
1 PITA, a pre-bilaterian p75NTR, is the evolutionary ancestor of TNF
}

6 Mark J. Cumming ${ }^{1,2}$, Julien Gibon ${ }^{2}$, Wayne S. Sossin ${ }^{1}$, Philip A. Barker ${ }^{2 *}$

$9{ }^{1}$ Department of Neurology and Neurosurgery, Montreal Neurological Institute, McGill

10 University, Montreal, Quebec, Canada

11

$12{ }^{2}$ Department of Biology, University of British Columbia Okanagan, Kelowna, British Columbia,

13 Canada

14

15

16

$17 *$ Corresponding author

18 E-mail: philip.barker@ubc.ca (PAB)

19

20

21 


\section{Abstract}

24 Tumor necrosis factor receptors (TNFRs) regulate a diverse array of biological functions,

25 including adaptive immunity, neurodevelopment, and many others. Although TNFRs are

26 expressed in all metazoan phyla, a coherent model of the molecular origins of mammalian

27 TNFRs — and how they relate to TNFRs in other phyla — has remained elusive. To address this,

28 we executed a large-scale, systematic Basic Local Alignment Search Tool (BLAST)-based

29 approach to trace the evolutionary ancestry of all 29 human TNFRs. We discovered that all

30 human TNFRs are descendants of a single pre-bilaterian TNFR with strong sequence similarity

31 to the p75 neurotrophin receptor (p75NTR), which we designate as PITA for ' $p 75 N T R$ is the

32 TNFR Ancestor'. A distinct subset of human TNFRs - including EDAR, XEDAR and TROY-

33 share a unique history as descendants of EDAR-XEDAR-TROY (EXT), which diverged from

34 PITA in a bilaterian ancestor. Most PITA descendants possess a death domain (DD) within their

35 intracellular domain (ICD) but EXTs do not. PITA descendants are expressed in all bilaterian

36 phyla and Cnidaria, but not in non-planulozoan ParaHoxozoa, suggesting that PITA originated in

37 an ancestral planulozoan. Drosophila melanogaster TNFRs (Wengen (Wgn) and Grindelwald

38 (Grnd)) were identified as divergent PITA descendants, providing the first evolutionary link

39 between this model TNFR system and the mammalian TNFR superfamily. This study reveals

40 PITA as the ancestor to human and Drosophila TNFR systems and describes an evolutionary

41 model that will facilitate deciphering TNF-TNFR functions in health and disease. 


\section{Introduction}

44 sarcoma by injection of a bacterial immunogen ('Coley toxin') directly into the primary tumor

45 (1). This anti-tumoral immune response was rediscovered 69 years later, with the discovery that sera derived from mice exposed to a bacterial polysaccharide could induce tumor regression in

47 vivo, suggesting that a 'tumor necrotizing factor' must be present in the serum (2). This factor was subsequently isolated and named tumour necrosis factor (TNF; now TNF $\alpha$ ) (3). Over the past decades, 19 human TNF ligands and 29 TNF receptors (TNFRs) have been characterized in humans [reviewed in (4)]. TNFRs mediate a diverse array of biological functions and are now known to function as critical regulators of innate immunity $(5,6)$; adaptive immunity $(7,8)$; and nervous system development (9-15) and maintenance (16-18). Aberrant TNFR signaling is implicated in viral infections (19), autoimmune disorders (20,21), neuropsychiatric disorders

54 (22-24), neurodegenerative disease (25-31), and other disease states $(32,33)$.

TNFRs are non-enzymatic single-pass transmembrane proteins that signal via liganddependent and -independent recruitment of adaptor proteins to their intracellular domain (ICD), most notably the TNFR-associated factor (TRAF) family of ubiquitin ligases (34). TNFRs are structurally related by the presence of one or more cysteine-rich domains (CRDs) in their

59 extracellular domain (ECD). The CRD is $\sim 40$ amino acids (AA) in length and contains 6 conserved cysteine residues, which form a specific pattern of disulfide bridging $\left(\mathrm{C}_{1}-\mathrm{C}_{2}, \mathrm{C}_{3}-\mathrm{C}_{5}\right.$,

$61 \mathrm{C}_{4}-\mathrm{C}_{6}$ ) that provide structural rigidity to the domain. Extracellular CRDs are typically arranged 62 in tandem arrays that produce distinct 'CRD clusters' - with an overall primary sequence unique 63 to each TNFR - that mediate ligand binding. Most TNFRs exist as homotrimers (35) and signal 64 in a 3:3 TNF:TNFR stoichiometry, with the exception of p75NTR. p75NTR does not bind a 
TNF ligand, but instead binds neurotrophin (NT) ligands (NGF, BDNF, NT-3 and NT-4/5) in a 2:2 NT:p75NTR stoichiometry (36). p75NTR also binds the pro-form of NGF if the Vps10p

\section{7} family member sortilin is present as a co-receptor $(37,38)$. NT-dependent and -independent signaling via p75NTR enables this receptor to regulate a broad array of nervous system signaling events $(39-41)$

A set of pro-apoptotic TNFRs — known as death receptors - possess an 80 amino acid intracellular death domain (DD) that can engage the extrinsic apoptotic pathway. Homotypic DD interactions with cytosolic death effectors initiate apoptotic signaling cascades that converge on mitochondrial permeabilization and executioner caspase activation $(4,42)$. p75NTR and death receptor 6 (DR6) are atypical DRs that mediate cell death via distinct signaling mechanisms $75(39,43)$.

Despite advances to our understanding of TNFR biology, our knowledge of TNFR evolution is limited. TNFRs have been identified in extant species of every animal phylum, but DRs are absent in choanoflagellates and Porifera, suggesting DRs may have originated from a gene fusion event between CRD and DD-containing proteins in an ancestral planulozoan $(44,45)$. CfTNFR — a DR expressed in the Zhikong scallop (Chlamys farreri)—shows primary sequence

81 homology to p75NTR in several vertebrate species (46), suggesting that mammalian TNFRs may

82 be descendants of an ancient DR. DRs with sequence homology to p75NTR and EDAR have

83 been discovered in sea anemone, sea urchin, annelids and tunicates (47-49). Moreover,

84 invertebrate chordates express several TNFRs with homology to p75NTR and EDAR, which

85 mediate development of the nervous system and epithelial appendages (44,50,51). Diverse TNFRs with sequence similarity to p75NTR have also been observed in platyhelminthes (52) In

87 Drosophila melanogaster, a TNF system that includes one form of TNF (Eiger) and 2 TNFRs 
88 [Wengen (Wgn) and Grindelwald (Grnd)] is being increasingly used as a model for TNF/TNFR

89 signaling (53-58). Though individual CRDs of these Drosophila TNFRs show homology to

90 several mammalian TNFRs $(53,56,58)$, their evolutionary link to the human TNFR system

91 remains uncertain.

Although TNFRs have been observed in all metazoan phyla $(44,45)$, a comprehensive

93 model of TNFR evolution describing the molecular origins of mammalian TNFRs — and how

94 they relate to the Drosophila model system — has remained elusive. To address this problem, we

95 traced the history of all human TNFRs in progressively older extant species spanning the animal

96 kingdom. Our evolutionary tracing focused specifically on the CRD cluster sequence of each

97 TNFR, since this site possesses strong selective pressure with sufficient sequence diversity to

98 distinguish between the receptors. This iterative BLAST strategy provided new insights into the

99 evolutionary history of human TNFRs and identified PITA — a pre-bilaterian TNFR with strong

100 sequence identity to p75NTR — as the common ancestor to mammalian TNFRs. This approach

101 also demonstrated that PITA is conserved all planulozoan phyla and gave rise to the divergent

102 Wgn-Grnd system, thereby establishing an evolutionary link between human TNFRs and

103 Drosophila counterparts. 


\section{Methods}

\section{Evolutionary tracing of human TNFRs}

Evolutionary tracing was performed using an iterative BLAST (Basic Local Alignment Search Tool) approach. The CRD cluster sequence for each TNFR was input into BLASTp, queried in the next most ancient extant species, and analyzed according to default algorithm parameters $($ expect threshold $=10 ;$ word size $=6 ;$ BLOSUM62 scoring matrix; gap costs existence: 11 extension: 1; application of a conditional compositional score matrix adjustment; and automatic input parameter adjustment for short sequences). No filtering or masking was applied to the BLASTp query. BLAST hits were identified as bona fide homologs if they met the following criteria: (i) E-value $\leq 0.01$; (ii) possess 1 or more TNFR CRDs; and (iii) single-pass transmembrane protein. If only 1 protein met these criteria, this protein was identified as a bona fide homolog, and the primary sequence that was homologous to the input (within the CRD cluster) was used as the input sequence for the subsequent BLASTp query in the next most ancient extant species. If 2 or more proteins met these criteria, the strongest bona fide homolog was identified by meeting these criteria: (i) full-length primary sequence length is most similar to that of the query protein; (ii) BLASTp querying the CRD cluster from other species consistently yields this homolog as the top hit; and (iii) lowest E-value. If no proteins met the basic criteria for homology, the analysis was repeated using the CRD cluster from previous species (as the BLASTp input) in the following order: (i) previous species expressing a homolog; (ii) Lingula anatina (if applicable); (iii) a mollusc expressing a homolog (if applicable); (iv) an arthropod expressing a homolog (if applicable); (v) an echinoderm expressing a homolog; (vi) a prevertebrate chordate and/or hemichordate expressing a homolog (if applicable); (vii) a teleost expressing a homolog (if applicable); then (viii) the human sequence. If no homolog is identified 
after these post hoc BLASTp queries, we concluded that no TNFR homolog exists in the queried species. If multiple accession numbers are identified for the same homolog, the accession number adhering to standard NCBI nomenclature (e.g. beginning with $\mathrm{NP}_{-}$or $\mathrm{XP}_{-}$) was selected.

\section{Identification of PITA, EXT, Wgn and Grnd family members in Protostomia}

The CRD cluster sequences PITA and EXT (from Limulus polyphemus), Wgn

(Drosophila melanogaster), and Grnd (Drosophila melanogaster) were input into BLASTp and queried in large protostomian taxa, including: Class Insecta, Class Crustacea, Class Chelicerata, Phylum Nematod), and Superphylum Lophotrochozoa (2nd outgroup). BLASTp queries were performed according to default algorithm parameters. Hits with an E-value $\leq 0.001$ were identified as bona fide homologs.

\section{Structural analysis of PITA descendants}

Full-length protein sequences for PITA descendants were analyzed for known domains and motifs using the NCBI Conserved Domains Database (CDD) and Pfam (59). Sequence alignments of PITA descandants with human p75NTR were performed in ClustalX version 2.1, and amino acid identity percentages from these alignment were analyzed in Clustal Omega (60). Reconstruction of the known PITA structures was performed manually in Inkscape Version 0.92.

\section{Evolutionary modelling}

All evolutionary modelling was performed using Randomized Axelerated Maximum Likelihood (RAxML) Version 8 software (61). Multiple sequence alignments input into RAxML 
bioRxiv preprint doi: https://doi.org/10.1101/2021.12.26.474206; this version posted December 26, 2021. The copyright holder for this preprint (which was not certified by peer review) is the author/funder, who has granted bioRxiv a license to display the preprint in perpetuity. It is made available under aCC-BY 4.0 International license.

151 were generated with ClustalX version 2.1. RAxML output trees were visualized using Interactive

152 Tree of Life (iTOL) version 6.3.2.

153

154 Graphing

155 Graphs were generated using GraphPad Prism v9.2.0.

156 


\section{Results}

158

\section{Human TNFRs are PITA descendants}

TNFRs are structurally identifiable by presence of one or more CRDs in their ECD (Table 1). Due the strong selective pressure on these CRD clusters - and the ability to distinguish TNFRs based on primary sequence variation within this region — evolutionary tracing was performed on the CRD cluster of human TNFRs by iterative BLAST queries in extant species with progressively older common ancestry (e.g. the CRD cluster sequence for human TNFR1 was queried in the non-human primate Pongo abelii; the homologous CRD region in P. abelii TNFR1 homolog was BLAST queried in the non-primate eutherian Mus musculus, and so on). Extant species chosen for evolutionary tracing: (i) were selected in an unbiased manner, (ii) span all animal phyla, and (iii) possess a fully sequenced genome (Table 2). As a negative control, the cysteine-rich region (CRR; also known as 'complement type repeat') of low-density lipoprotein receptor (LDLR) family members was traced in parallel (S1 Table) due to high sequence similarity to TNFR CRD clusters. The longest tandem cluster of CRRs for a given LDLR family member was selected for evolutionary analysis (S1 Table); importantly, LDLR evolutionary tracing showed no overlap with TNFR evolution.

Table 1. Human TNFRs and their CRD clusters used in evolutionary analyses.

\begin{tabular}{cc|cc}
\hline & TNF Receptor & \multicolumn{2}{c}{ CRD Cluster } \\
\hline Abbreviation & Protein Name (Standardized Name) & \#CRDs & AA \\
\hline 4-1BB & 4-1BB $($ TNFRSF9) & 4 & $24-159$ \\
BAFFR & B cell-activating factor receptor $($ TNFRSF 13) & 1 (truncated) & $18-35$ \\
BCMA & B cell maturation antigen $($ TNFRSF17) & 1 & $7-41$ \\
CD27 & CD27 antigen $(T N F R S F 7)$ & 3 & $26-141$ \\
CD30 & CD30 antigen $($ TNFRSF8) & 6 & $28-325$ \\
CD40 & CD40 antigen $($ TNFRSF5) & 4 & $25-187$ \\
DcR1 & Decoy receptor 1 (TNFRSF10C) & 3 & $29-149$ \\
DcR2 & Decoy receptor 2 (TNFRSF10D) & 3 & $58-180$ \\
DcR3 & Decoy receptor 3 (TNFRSF6B) & 4 & $31-193$
\end{tabular}




\begin{tabular}{|c|c|c|c|}
\hline DR3 & Death receptor 3 (TNFRSF25) & 4 & $34-192$ \\
\hline DR4 & Death receptor 4 (TNFRSF10A) & 3 & $107-229$ \\
\hline DR5 & Death receptor 5 (TNFRSF1OB) & 3 & $57-178$ \\
\hline DR6 & Death receptor $6(T N F R S F 21)$ & 4 & $50-211$ \\
\hline EDAR & Ectodysplasin A receptor $(E D A R)$ & 3 & $30-148$ \\
\hline Fas & Fas receptor (TNFRSF6) & 3 & $47-166$ \\
\hline Fn14 & TWEAK receptor (TNFRSF12A) & 1 (atypical) & $36-67$ \\
\hline GITR & $\begin{array}{l}\text { Glucocorticoid-induced TNFR-related gene } \\
\qquad(T N F R S F 18)\end{array}$ & 3 & $34-153$ \\
\hline HVEM & Herpesvirus entry mediator (TNFRSF 14) & 3 & $42-162$ \\
\hline LT $\beta R$ & Lymphotoxin B receptor (TNFRSF3) & 4 & $42-211$ \\
\hline OPG & Osteoprotegerin $(T N F R S F 11 B)$ & 4 & $24-185$ \\
\hline OX40 & OX40 antigen (TNFRSF4) & 4 & $30-167$ \\
\hline p75NTR & p75 neurotrophin receptor (TNFRSF16) & 4 & $31-188$ \\
\hline RANK & $\begin{array}{l}\text { Receptor activator of nuclear factor } \mathrm{kB} \\
\qquad(T N F R S F 11 A) \\
\text { Receptor expressed in lymphoid tissues }\end{array}$ & 4 & $34-194$ \\
\hline RELT & & 2 & $28-90$ \\
\hline TACI & $\begin{array}{l}\text { Iransmembrane activator and CAIVIL interactor } \\
\qquad(T N F R S F 13 B)\end{array}$ & 2 & $33-104$ \\
\hline TNFR1 & Tumor necrosis factor receptor 1 (TNFRSF 1A) & 4 & $43-196$ \\
\hline TNFR2 & $\begin{array}{c}\text { Tumor necrosis factor receptor } 2(T N F R S F 1 B) \\
\text { Tumor necrosis factor receptor orphan Y }\end{array}$ & 4 & $39-201$ \\
\hline TROY & $(T N F R S F 19)$ & 3 & $33-149$ \\
\hline XEDAR & $\mathrm{X}$-linked ectodysplasin receptor $(X E D A R)$ & 3 & 2-118 \\
\hline
\end{tabular}

175 Table 2. Species used in human TNFR evolutionary analysis

\begin{tabular}{cc|cc}
\hline Linnaean Name & Common Name & Phylum & Subtaxon \\
\hline Homo sapiens & Human & Chordata & Primates \\
Pongo abelii & Sumatran orangutan & Chordata & Primates \\
Mus musculus & House mouse & Chordata & Eutheria \\
Ornithorhynchus anatinus & Platypus & Chordata & Mammalia \\
Gallus gallus & Chicken & Chordata & Amniota \\
Xenopus tropicalis & Tropical clawed frog & Chordata & Tetrapoda \\
Latimeria chalumnae & Coelacanth & Chordata & Vertebrata \\
Danio rerio & Zebrafish & Chordata & Vertebrata \\
& Florida lancelet & & \\
Branchiostoma floridae & (Amphioxus) & Chordata & Cephalochordata \\
Saccoglossus kowalevskii & Acorn worm & Hemichordata & - \\
Strongylocentrotus purpuratus & Purple sea urchin & Echinodermata & -
\end{tabular}


Acyrthosiphon pisum

Drosophila melanogaster

Daphnia pulex

Daphnia magna

Limulus polyphemus

Dermatophagoides pteronyssinus

Caenorhabditis elegans

Helobdella robusta

Capitella teleta

Crassostrea gigas

Octopus bimaculoides

Schmidtea mediterranea

Lingula anatina

Nematostella vectensis

Hydra vulgaris

Trichoplax adhaerens

Amphimedon queenslandica

Mnemiopsis leidyi
Pea aphid

Fruit fly

Common water flea

Water flea

Atlantic horseshoe crab

European house dust mite

Roundworm

Californian leech

Segmented worm

Pacific oyster

California two-spot octopus

Freshwater planarian flatworm

Oriental lamp shell

Starlet sea anemone

Swiftwater hydra N/A

Sponge

Sea walnut
Arthropoda

Insecta

Arthropoda

Insecta

Arthropoda

Crustacea

Arthropoda

Crustacea

Arthropoda

Chelicerata

Arthropoda

Chelicerata

Nematoda

Annelida

Annelida

Mollusca

Mollusca

Gastropoda

Cephalopoda

Platyhelminthes

Brachiopoda

Cnidaria

Cnidaria

Placozoa

Porifera

Ctenophora
176

177

178

179

180

181

182

183

184

185

186

187

Figure 1. Human TNFRs are PITA descendants. (A) Evolutionary tracing of all 29 human

TNFRs. Each dot represents a single homologous protein. A solid outline denotes that the homolog is a TNFR [(i) possesses 1 or more CRDs + a single TMD, and (ii) does not non-CRD functional domains in the ECD]. A dashed outline indicates the protein is not a TNFR [violates criterion (i) and/or (ii)]. The dot's fill denotes the domains that can recapitulate that homolog (black - a non-p75NTR CRD, green - p75NTR CRD and/or the p75NTR DD and/or the p75NTR TMD-Chopper region, cyan - p75NTR CRD only, pink - p75NTR DD only). (B)

Schematic structure of human p75NTR and the domains/motifs/residues analyzed for homology in PITA descendants. (C-H) Structure and \% AA identity to human p75NTR (by domain) of PITA descendants expressed in: (C) swiftwater hydra (Hydra vulgaris), (D) starlet sea anemone (Nematostella vectensis), (E) brachiopod (Lingula anatina), (F) Pacific oyster (Crassostrea 
gigas), (G) segmented worm (Capitella teleta), and (H) European house dust mite

(Dermatophagoides pteronyssinus).

Remarkably, the ancestry of 25 human TNFRs could be traced to 2 TNFRs expressed in the

purple sea urchin (Strongylocentrotus purpuratus) (Fig 1A; S1 Table). Of the 25 human TNFRs analyzed,

19322 share a common ancestry to a S. purpuratus TNFR (XP_030830229.1) that contains 4 CRDs and a

194 death domain. Further tracing of this $S$. purpuratus receptor revealed that orthologs are expressed in a 195 wide array of animal phyla, including Arthropoda, Annelida, Mollusca, Brachiopoda, and Cnidaria (Fig 196 1A).

The other 3 human TNFRs (EDAR, XEDAR, and TROY) were related to a distinct S. purpuratus homolog of this $S$. purpuratus TNFR in an arthropod (Atlantic horseshoe crab) but no other orthologs could be identified in lophotrochozoan or pre-bilaterian phyla (Fig 1A). This indicates that the ancestor bilaterian and pre-bilaterian phyla. because these family members possess 1-2 CRDs that limit the statistical power that applied to their 209 evolutionary tracing. It seems likely that these 4 TNFRs share ancestry to at least 1 of the 2 s ancestral

210 TNFRs expressed in S. purpuratus — as is the case for the other 25 human TNFRs — but this remains 211 unconfirmed. 
We next sought to identify the human TNFR that most closely resembles the sole ancestor

214 protostomian and Cnidarian species was BLAST queried in Homo sapiens. Reverse BLAST analyses

215 identified p75NTR $\left(10^{-9}<\mathrm{E}<10^{-48}\right)$ as the most significant hit (lowest E-value) for every ancestral TNFR

216 sequence queried (Table 3). To corroborate the reverse BLAST result, we ran evolutionary traces on

217 additional p75NTR domains, including the: (i) stalk domain, (ii) transmembrane and chopper domains

218 (TMD-Chopper), and (iii) death domain (DD). Evolutionary tracing of p75NTR by the TMD-Chopper

219 and DD domains replicated the p75NTR ancestry resolved using the CRD cluster analysis (Fig 1A; S1

220 Table). This demonstrates that p75NTR retained the structural characteristics of the ancestral TNFR.

221 Therefore, we designate the ancestral TNFR PITA, for $\boldsymbol{p} 75 N T R$ is the TNFR Ancestor.

227 intracellular juxtamembrane domain $(19.15 \% \leq$ AA identity $\leq 31.11 \%)$ and extracellular stalk domain $(14.52 \% \leq$ AA identity $\leq 30.43 \%)($ Figs 1C-I; S2 Table).

PITA likely arose from a gene fusion event. To identify extant species carrying domains that contributed to PITA, we traced the p75NTR CRD cluster in pre-ParaHoxozoan phyla using the iterative BLAST strategy. This analysis identified XP_019851134.1 in the Poriferan Amphimedon queenslandica

232 (Great Barrier Reef sponge) as a potential origin of the PITA CRD cluster (Fig 1A). Sequence alignment 233 of the CRD clusters from human p75NTR and XP_019851134.1 revealed strong sequence homology

234 (28.32\% AA identity) (S3 Fig). A reverse BLAST query of full-length XP_019851134.1 in Homo sapiens 235 revealed no human ortholog, but the existence of such an ortholog cannot be ruled out.

236 Similar evolutionary tracing of the p75NTR DD identified XP_019852665.1 in A. queenslandica 237 as a potential origin of the PITA DD (Fig 1A). A reverse BLAST query of full-length XP_019852665.1 in 
Homo sapiens identified this PITA DD ancestor as an ortholog of Death-Associated Protein Kinase 1 of genomic loci encoding the XP_019851134.1 CRD cluster and DAPK1 DD occurred in an ancestral planulozoan. Timetree estimates (62) indicate that this putative fusion event occurred 650-750 million

244 years ago.

Based on the cumulative evidence, we conclude that PITA is the evolutionary ancestor of the

246 human TNFR superfamily. p75NTR retained the ancient PITA structure and all other TNFRs diverged

247 from PITA to acquire unique biological functions. We propose that PITA originated in an ancestral

248 planulozoan from a genomic fusion event between sequences encoding a CRD cluster (XP_019852665.1) and the DD of DAPK1.

\section{PITAs and EXTs are independent TNFR families}

Although all human TNFRs are PITA descendants, EDAR, XEDAR and TROY are descendants of EXT, which diverged in an ancestral bilaterian, earlier than the PITA divergence events in early chordates (Fig 1A). To test if PITAs and EXTs are independent TNFR families, we generated the maximum likelihood phylogeny between PITAs and EXTs (from protostome and deuterostome species) using RAxML. Wgn and Grnd were incorporated into the analysis to clarify the evolutionary relationship

257 between PITAs/EXTs and the Drosophila TNFR model system. To identify PITA and EXT homologs

258 across bilaterian taxa, we performed a large-scale BLAST search using the L. polyphemus CRD clusters

259 for PITA and EXT as query sequences (S5 Table). We repeated this strategy to identify Wgn and Grnd

260 homologs by BLAST queries of the D. melanogaster CRD cluster sequences with Protostomia. Maximum

261 likelihood modelling of CRD clusters demonstrated that PITAs and EXTs are independent protein

262 families. Importantly, human and murine p75NTR were confirmed as PITA family members, whereas 
EDAR, XEDAR and TROY were confirmed as EXT family members (Fig. 2A). This indicates that a PITA duplication event in an ancestral bilaterian generated independent PITA and EXT families of TNFRs. In humans, EDAR, XEDAR and TROY are classified as EXT family members; all other TNFRs are classified as PITAs.

Figure 2. PITAs and EXTs are independent TNFR families. (A) RAxML evolutionary modelling of CRD cluster sequences from PITAs, EXTs, and Wgn/Grnd homologs spanning protostome and deuterostome phyla. The CRR cluster sequence from L. polyphemus LDLR was used as an outgroup. (B) Detection of DD in all identified PITA and EXT homologs. Graph

272 represents percentage of protostome PITAs and EXTs where a DD is present (DR) or absent

273 (non-DR). DD search cross-referenced both the NCBI Protein and Pfam databases. (C)

274 Percentage of all non-DR PITAs expressed in species that express at least one DR PITA. 'Yes'

275 indicates the non-DR host species expresses a DR PITA; 'No' indicates the non-DR host species 276 does not express a DR PITA; and "N/A" indicates the host species' PITA homologs are only 277 partially sequenced, therefore no determination could be made. EXT CRD cluster sequences to identify all protostome homologs. For each identified homolog, we crossreferenced the NCBI Protein and PFAM (63) databases for the presence of a DD (S6 Table). DD-positive homologs were classified as DRs, and DD-negative homologs were identified as non-DRs. Figure 2B shows that $43.5 \%$ of all PITAs are DRs, whereas only $3.4 \%$ of EXTs possess a DD. To clarify the DR status of the PITA family, we measured the proportion of non-DR PITAs expressed in organisms 
PITA (Fig 2C). These data collectively establish that the vast majority of DRs are PITA descendants, but that rare EXT-descendant DRs also appear in nature (e.g. EDAR in chordates).

\section{Wengen and Grindelwald are divergent PITA descendants}

Wgn and Grnd represent a simple TNFR system assumed to be homologous to mammalian

TNFRs. However, the human TNFR evolutionary analysis described in Figure 1 failed to show structural

293 homology of any human TNFR to any protein in the D. melanogaster proteome. Given that Wgn/Grnd

294 families are more similar to PITAs than EXTs (Fig 2A), a potential explanation is Wgn and Grnd are divergent PITA descendants that lack direct homology to a human TNFR.

To test this hypothesis, we ran large-scale BLAST queries of CRD clusters from Wgn, Grnd and

TNFR expressed in Amphibalanus amphitrite (Acorn barnacle) showing weak homology to both 
311 Grnd-possess a TNFR CRD consensus sequence (45), ruling out the possibility that an

312 unknown non-PITA TNFR gave rise to the Wgn-Grnd system. This finding, in conjunction with

313 our analyses described above (Fig 2A; Fig 3), indicate that Wgn and Grnd are divergent PITA

314 descendants that share a common evolutionary ancestor with human TNFRs.

Figure 3. Drosophila TNFRs Wengen and Grindelwald are divergent PITA descendants.

317 (A) Phylogenetic reconstruction of all ecdysozoan species expressing Wgn, Grnd and/or PITA sequences for Wgn and Grnd; Limulus polyphemus sequence for PITA). Each species’ TNFR expression profile is represented by the bar colour next to the species name (green - PITA only,

321 red - Wgn only, blue - Grnd only, magenta - Wgn + Grnd, yellow - PITA + Wgn, cyan - PITA

$322+$ Grnd, black - Wgn + Grnd + PITA). E-value $\leq 0.01$ denotes a bona fide homolog. (B) Venn

323 diagram summary of the TNFR expression profiles of ecdysoa shown in (A). Lophotrochozoa

324 are included as an outgroup.

Finally, within arthropods, an unexpected trend in the species distribution of PITA and

327 Wgn/Grnd homologs was observed between Diptera and Hymenoptera. Specifically, Dipteran

328 species, including D. melanogaster, exclusively express a Wgn/Grnd or Wgn-only TNFR system

329 (no PITAs) whereas Hymenopteran species express a PITA-only TNFR system (no Wgn or

330 Grnd). This suggests that selective pressure may result in mutually exclusive expression of these

331 TNFR systems in Diptera and Hymenoptera.

Based on the available evidence, we propose the following model of $\mathrm{Wgn} / \mathrm{Grnd}$ 
334 Grnd originated from a Wgn gene duplication in a common ancestor to insects and crustaceans;

335 and (iii) Wgn and Grnd diverged from PITA to acquire function as receptors to the TNF ligand

336 Eiger.

Discussion

human TNFRs by tracing the origin of the TNFR superfamily to a single ancestral receptor,

341 PITA, that possesses remarkable sequence homology to p75NTR. p75NTR-PITA sequence

342 homology is strongest within the CRD and DD clusters, suggesting that p75NTR and PITA may

343 bind related ligand(s) and activate similar death domain-mediated signaling events. PITA likely

344 originated from a genomic fusion event between the CRD cluster of an uncharacterized TNFR

345 (ancestral to XP_019851134.1 in Amphimedon queenslandica) and the DD of DAPK1 in an

346 ancestral planulozoan. According to the Time Tree of Life database (62), the most recent

347 common ancestor between the Homo and Nematostella genera existed between 647 and 747

348 million years ago, thus providing an estimate of when this ancestral TNFR originated. PITA is

349 strongly conserved within bilaterian and Cnidarian taxa, indicating it plays a critical

350 physiological role in these species.

Divergence of the PITA lineage occurred first in an ancestral bilaterian, which generated

352 the EXT ancestor; and second, during a massive TNFR expansion event in early chordates. The

353 former event gave rise to an independent TNFR family— known as the EXT family—that

354 proliferated across bilaterian phyla. The latter event coincides with emergence of the adaptive

355 immune system which relies on diverse TNFR signaling events (7). Domain analysis 
demonstrated that PITAs are commonly death receptors, but EXTs are not, though rare EXTdescendant death receptors do exist. In humans, the closest PITA relative, p75NTR, is a death receptor but most human PITA descendants have lost their intracellular DD. In protostomes, non-DD PITA descendants primarily arise in species already expressing a PITA death receptor. EDAR, XEDAR and TROY are the only EXT descendants expressed in humans. Since non-DR EXTs are prevalent in protostomes, we assume EDAR must have acquired its intracellular DD

362 following an EXT gene duplication event in an early chordate/vertebrate. We suspect the ancestor to human EXTs must have closely resembled XEDAR or TROY, but this is not definitive; neither receptor shows strong conservation to the ancestral sequence, as is observed with p75NTR and ancestral PITA. sea anemones. Morphological features appearing in Cnidaria include tissue layering, radial symmetry, and synaptic neural networks (64). Importantly, the placozoan Trichoplax adhaerens — which lacks adaptations that lead to fast synaptic transmission (e.g. synaptic cleft,

371 synaptic vesicles, etc.) (65)—does not express a PITA descendant. Thus, PITA expression may

372 be limited to animals possessing synaptic neural networks. This in turn suggests that the

373 emergence of PITA may have been a prerequisite for the development of complex nervous

374 system networks. Future investigation into the biological function of PITA in a Cnidarian model 375 organism, such as the sea anemone Nematostella vectensis, may yield insight into the core 376 functionality of this TNFR ancestor. 


\section{Conclusions}

381 identified the death receptor PITA — a novel TNFR with strong sequence homology to

382 p75NTR - as the molecular ancestor to the mammalian TNFR superfamily. PITA was likely

383 created by the fusion of genomic loci encoding the CRD cluster of the uncharacterized TNFR

384 XP_030830229.1 and the DD of DAPK1 in a common ancestor to humans and the demosponge

385 Amphimedon queenslandica. The PITA receptor has remained structurally intact over 750

386 million years of evolution and gave rise to several descendent TNFR families in deuterostomes

387 and protostomes, including the Drosophila melanogaster TNFRs Wgn and Grnd. 


\section{References}

399 1. Coley WB. The treatment of malignant tumors by repeated innoculations of erysipelas: with a

400 report of ten original cases. American Journal of the Medical Sciences. 1893;10:487-511.

4012 2. O'malley WE, Achinstein B, Shear MJ. ACTION OF BACTERIAL POLYSACCHARIDE ON TUMORS. III. 402 REPEATED RESPONSE OF SARCOMA 37, IN TOLERANT MICE, TO SERRATIA MARCESCENS ENDOTOXIN. 403 Cancer Res. 1963 Jul;23:890-5.

404 3. Carswell EA, Old L, Kassel RL, Green S, Fiore N, Williamson B. An endotoxin-induced serum 405 factor that causes necrosis of tumors. Proc Natl Acad Sci USA. 1975 Sep;72(9):3666-70.

406 4. Aggarwal BB, Gupta SC, Kim JH. Historical perspectives on tumor necrosis factor and its 407 superfamily: 25 years later, a golden journey. Blood. 2012 Jan 19;119(3):651-65.

408 5. Francisco NM, Hsu N-J, Keeton R, Randall P, Sebesho B, Allie N, et al. TNF-dependent regulation 409 and activation of innate immune cells are essential for host protection against cerebral tuberculosis. J 410 Neuroinflammation. 2015 Jun 26;12:125.

411 6. Segueni N, Benmerzoug S, Rose S, Gauthier A, Bourigault M-L, Reverchon F, et al. Innate myeloid 412 cell TNFR1 mediates first line defence against primary Mycobacterium tuberculosis infection. Sci Rep. 4132016 Mar 2;6:22454.

$4147 . \quad$ Ward-Kavanagh LK, Lin WW, Šedý JR, Ware CF. The TNF Receptor Superfamily in Co-stimulating 415 and Co-inhibitory Responses. Immunity. 2016 17;44(5):1005-19.

$416 \quad$ 8. Bittner S, Knoll G, Ehrenschwender M. Death receptor 3 signaling enhances proliferation of 417 human regulatory T cells. FEBS Lett. 2017;591(8):1187-95.

418 9. Yeo TT, Chua-Couzens J, Butcher LL, Bredesen DE, Cooper JD, Valletta JS, et al. Absence of 419 p75NTR causes increased basal forebrain cholinergic neuron size, choline acetyltransferase activity, and 420 target innervation. J Neurosci. 1997 Oct 15;17(20):7594-605.

421 10. Bamji SX, Majdan M, Pozniak CD, Belliveau DJ, Aloyz R, Kohn J, et al. The p75 neurotrophin 422 receptor mediates neuronal apoptosis and is essential for naturally occurring sympathetic neuron death. 423 J Cell Biol. 1998 Feb 23;140(4):911-23.

424 11. O'Keeffe GW, Gutierrez H, Pandolfi PP, Riccardi C, Davies AM. NGF-promoted axon growth and 425 target innervation requires GITRL-GITR signaling. Nat Neurosci. 2008 Feb;11(2):135-42.

426 12. Park KJ, Grosso CA, Aubert I, Kaplan DR, Miller FD. p75NTR-dependent, myelin-mediated axonal 427 degeneration regulates neural connectivity in the adult brain. Nat Neurosci. 2010 May;13(5):559-66.

428 13. Tam SJ, Richmond DL, Kaminker JS, Modrusan Z, Martin-McNulty B, Cao TC, et al. Death 429 receptors DR6 and TROY regulate brain vascular development. Dev Cell. 2012 Feb 14;22(2):403-17.

$430 \quad 14 . \quad K i s i s w a$ L, Osório C, Erice C, Vizard T, Wyatt S, Davies AM. TNFa reverse signaling promotes 431 sympathetic axon growth and target innervation. Nat Neurosci. 2013 Jul;16(7):865-73. 
15. Olsen O, Kallop DY, McLaughlin T, Huntwork-Rodriguez S, Wu Z, Duggan CD, et al. Genetic analysis reveals that amyloid precursor protein and death receptor 6 function in the same pathway to control axonal pruning independent of $\beta$-secretase. J Neurosci. 2014 May 7;34(19):6438-47.

16. Rösch H, Schweigreiter R, Bonhoeffer T, Barde Y-A, Korte M. The neurotrophin receptor p75NTR modulates long-term depression and regulates the expression of AMPA receptor subunits in the hippocampus. Proc Natl Acad Sci USA. 2005 May 17;102(20):7362-7.

17. Woo NH, Teng HK, Siao C-J, Chiaruttini C, Pang PT, Milner TA, et al. Activation of p75NTR by proBDNF facilitates hippocampal long-term depression. Nat Neurosci. 2005 Aug;8(8):1069-77.

18. Marik SA, Olsen O, Tessier-Lavigne M, Gilbert CD. Death receptor 6 regulates adult experiencedependent cortical plasticity. J Neurosci. 2013 Sep 18;33(38):14998-5003.

19. Wortzman ME, Clouthier DL, McPherson AJ, Lin GHY, Watts TH. The contextual role of TNFR family members in CD8(+) T-cell control of viral infections. Immunol Rev. 2013 Sep;255(1):125-48.

20. Dostert C, Grusdat M, Letellier E, Brenner D. The TNF Family of Ligands and Receptors: Communication Modules in the Immune System and Beyond. Physiol Rev. 2019 01;99(1):115-60.

21. Jarosz-Griffiths HH, Holbrook J, Lara-Reyna S, McDermott MF. TNF receptor signalling in autoinflammatory diseases. Int Immunol. 2019 Sep 18;31(10):639-48.

22. Fujii T, Kunugi H. p75NTR as a therapeutic target for neuropsychiatric diseases. Curr Mol Pharmacol. 2009 Jan;2(1):70-6.

23. Buchhave P, Zetterberg H, Blennow K, Minthon L, Janciauskiene S, Hansson O. Soluble TNF receptors are associated with $A \beta$ metabolism and conversion to dementia in subjects with mild cognitive impairment. Neurobiol Aging. 2010 Nov;31(11):1877-84.

24. Puschban Z, Sah A, Grutsch I, Singewald N, Dechant G. Reduced Anxiety-Like Behavior and Altered Hippocampal Morphology in Female p75NTR(exon IV-/-) Mice. Front Behav Neurosci. 2016;10:103.

25. Perry RT, Collins JS, Wiener H, Acton R, Go RC. The role of TNF and its receptors in Alzheimer's disease. Neurobiol Aging. 2001 Dec;22(6):873-83.

26. Hu Y, Lee X, Shao Z, Apicco D, Huang G, Gong BJ, et al. A DR6/p75(NTR) complex is responsible for $\beta$-amyloid-induced cortical neuron death. Cell Death Dis. 2013 Apr 4;4:e579.

27. Xu Y, Wang D, Luo Y, Li W, Shan Y, Tan X, et al. Beta amyloid-induced upregulation of death receptor 6 accelerates the toxic effect of $\mathrm{N}$-terminal fragment of amyloid precursor protein. Neurobiol Aging. 2015 Jan;36(1):157-68.

28. Bachis A, Wenzel E, Boelk A, Becker J, Mocchetti I. The neurotrophin receptor p75 mediates gp120-induced loss of synaptic spines in aging mice. Neurobiol Aging. 2016;46:160-8.

29. Simmons DA, Belichenko NP, Ford EC, Semaan S, Monbureau M, Aiyaswamy S, et al. A small molecule p75NTR ligand normalizes signalling and reduces Huntington's disease phenotypes in R6/2 and BACHD mice. Hum Mol Genet. 2016 15;25(22):4920-38. 
30. Wang Y-R, Wang J, Liu Y-H, Hu G-L, Gao C-Y, Wang Y-J, et al. Cysteine-Rich Repeat Domains 2 and 4 are Amyloid- $\beta$ Binding Domains of Neurotrophin Receptor p75NTR and Potential Targets to Block Amyloid- $\beta$ Neurotoxicity. J Alzheimers Dis. 2018;63(1):139-47.

31. Patnaik A, Zagrebelsky M, Korte M, Holz A. Signaling via the p75 neurotrophin receptor facilitates amyloid- $\beta$-induced dendritic spine pathology. Sci Rep. 2020 Aug 7;10(1):13322.

32. Holbrook J, Lara-Reyna S, Jarosz-Griffiths H, McDermott M. Tumour necrosis factor signalling in health and disease. F1000Res. 2019;8.

33. So T, Ishii N. The TNF-TNFR Family of Co-signal Molecules. Adv Exp Med Biol. 2019;1189:53-84.

34. Park HH. Structure of TRAF Family: Current Understanding of Receptor Recognition. Front Immunol. 2018;9:1999.

35. Chan FK, Chun HJ, Zheng L, Siegel RM, Bui KL, Lenardo MJ. A domain in TNF receptors that mediates ligand-independent receptor assembly and signaling. Science. 2000 Jun 30;288(5475):2351-4.

36. Gong $\mathrm{Y}, \mathrm{Cao} \mathrm{P}, \mathrm{Yu} \mathrm{H}$, Jiang T. Crystal structure of the neurotrophin-3 and p75NTR symmetrical complex. Nature. 2008 Aug 7;454(7205):789-93.

37. Nykjaer A, Lee R, Teng KK, Jansen P, Madsen P, Nielsen MS, et al. Sortilin is essential for proNGFinduced neuronal cell death. Nature. 2004 Feb 26;427(6977):843-8.

38. Teng HK, Teng KK, Lee R, Wright S, Tevar S, Almeida RD, et al. ProBDNF induces neuronal apoptosis via activation of a receptor complex of p75NTR and sortilin. J Neurosci. 2005 Jun 1;25(22):5455-63.

39. Roux PP, Barker PA. Neurotrophin signaling through the p75 neurotrophin receptor. Prog Neurobiol. 2002 Jun;67(3):203-33.

40. Hempstead BL. The many faces of p75NTR. Curr Opin Neurobiol. 2002 Jun;12(3):260-7.

41. Schor NF. The p75 neurotrophin receptor in human development and disease. Prog Neurobiol. 2005 Oct;77(3):201-14.

42. Lavrik IN. Systems biology of death receptor networks: live and let die. Cell Death Dis. 2014 May 29;5:e1259.

43. Strilic B, Yang L, Albarrán-Juárez J, Wachsmuth L, Han K, Müller UC, et al. Tumour-cell-induced endothelial cell necroptosis via death receptor 6 promotes metastasis. Nature. 2016 11;536(7615):2158.

44. Wiens GD, Glenney GW. Origin and evolution of TNF and TNF receptor superfamilies. Dev Comp Immunol. 2011 Dec;35(12):1324-35.

45. Quistad SD, Traylor-Knowles N. Precambrian origins of the TNFR superfamily. Cell Death Discov. 2016 Jul 18;2:16058. 
501

502

503

504

505

506

507

508

509

510

511

512

513

514

515

516

517

518

519

520

521

522

523

524

525

526

527

528

529

530

531

532

533

534

535

536

537

46. Li L, Qiu L, Song L, Song X, Zhao J, Wang L, et al. First molluscan TNFR homologue in Zhikong scallop: molecular characterization and expression analysis. Fish Shellfish Immunol. 2009 Nov;27(5):62532.

47. Robertson AJ, Croce J, Carbonneau S, Voronina E, Miranda E, McClay DR, et al. The genomic underpinnings of apoptosis in Strongylocentrotus purpuratus. Dev Biol. 2006 Dec 1;300(1):321-34.

48. Pantalacci S, Chaumot A, Benoît G, Sadier A, Delsuc F, Douzery EJP, et al. Conserved features and evolutionary shifts of the EDA signaling pathway involved in vertebrate skin appendage development. Mol Biol Evol. 2008 May;25(5):912-28.

49. Lauri A, Bertucci P, Arendt D. Neurotrophin, p75, and Trk Signaling Module in the Developing Nervous System of the Marine Annelid Platynereis dumerilii. Biomed Res Int. 2016;2016:2456062.

50. Bridgham JT, Bobe J, Goetz FW, Johnson AL. Conservation of death receptor- 6 in avian and piscine vertebrates. Biochem Biophys Res Commun. 2001 Jun 29;284(5):1109-15.

51. Colosimo PF, Hosemann KE, Balabhadra S, Villarreal G, Dickson M, Grimwood J, et al. Widespread parallel evolution in sticklebacks by repeated fixation of Ectodysplasin alleles. Science. 2005 Mar 25;307(5717):1928-33.

52. Bertevello CR, Russo BRA, Tahira AC, Lopes-Junior EH, DeMarco R, Oliveira KC. The evolution of TNF signaling in platyhelminths suggests the cooptation of TNF receptor in the host-parasite interplay. Parasit Vectors. 2020 Sep 25;13(1):491.

53. Kanda H, Igaki T, Kanuka H, Yagi T, Miura M. Wengen, a member of the Drosophila tumor necrosis factor receptor superfamily, is required for Eiger signaling. J Biol Chem. 2002 Aug 9;277(32):28372-5.

54. Kauppila S, Maaty WSA, Chen P, Tomar RS, Eby MT, Chapo J, et al. Eiger and its receptor, Wengen, comprise a TNF-like system in Drosophila. Oncogene. 2003 Jul 31;22(31):4860-7.

55. Ruan W, Unsain N, Desbarats J, Fon EA, Barker PA. Wengen, the sole tumour necrosis factor receptor in Drosophila, collaborates with moesin to control photoreceptor axon targeting during development. PLoS ONE. 2013;8(3):e60091.

56. Andersen DS, Colombani J, Palmerini V, Chakrabandhu K, Boone E, Röthlisberger M, et al. The Drosophila TNF receptor Grindelwald couples loss of cell polarity and neoplastic growth. Nature. 2015 Jun 25;522(7557):482-6.

57. Ratheesh A, Biebl J, Vesela J, Smutny M, Papusheva E, Krens SFG, et al. Drosophila TNF Modulates Tissue Tension in the Embryo to Facilitate Macrophage Invasive Migration. Dev Cell. 2018 07;45(3):331-346.e7.

58. Palmerini V, Monzani S, Laurichesse Q, Loudhaief R, Mari S, Cecatiello V, et al. Drosophila TNFRs Grindelwald and Wengen bind Eiger with different affinities and promote distinct cellular functions. Nat Commun. 2021 Apr 6;12(1):2070.

59. El-Gebali S, Mistry J, Bateman A, Eddy SR, Luciani A, Potter SC, et al. The Pfam protein families database in 2019. Nucleic Acids Res. 2019 Jan 8;47(D1):D427-32. 
538 60. Madeira F, Park YM, Lee J, Buso N, Gur T, Madhusoodanan N, et al. The EMBL-EBI search and 539 sequence analysis tools APIs in 2019. Nucleic Acids Res. 2019 02;47(W1):W636-41.

540 61. Stamatakis A. RAxML version 8: a tool for phylogenetic analysis and post-analysis of large 541 phylogenies. Bioinformatics. 2014 May 1;30(9):1312-3.

542 62. Kumar S, Stecher G, Suleski M, Hedges SB. TimeTree: A Resource for Timelines, Timetrees, and 543 Divergence Times. Mol Biol Evol. 2017 01;34(7):1812-9.

544 63. Mistry J, Chuguransky S, Williams L, Qureshi M, Salazar GA, Sonnhammer ELL, et al. Pfam: The 545 protein families database in 2021. Nucleic Acids Res. 2021 Jan 8;49(D1):D412-9.

546 64. Watanabe H, Fujisawa T, Holstein TW. Cnidarians and the evolutionary origin of the nervous 547 system. Dev Growth Differ. 2009 Apr;51(3):167-83.

548 65. Smith CL, Varoqueaux F, Kittelmann M, Azzam RN, Cooper B, Winters CA, et al. Novel cell types, 549 neurosecretory cells, and body plan of the early-diverging metazoan Trichoplax adhaerens. Curr Biol. 5502014 Jul 21;24(14):1565-72. 


\section{Supplemental Information}

553 S1 Table. Raw TNFR and LDLR evolution data. The NCBI accession number and

554 homologous domain sequence for each identified TNFR/LDLR homolog.

555 S2 Table. Sequence identity of PITA descendants to human p75NTR by protein region.

556 S3 Figure. Structure of XP_019851134.1 in Amphimedon queenslandica. (A) Structural

557 schematic of A. queenslandica protein XP_019851134.1 (shown to scale). (B) Multiple sequence

558 alignment of the CRD cluster sequences for XP_019851134.1 and human p75NTR.

559 S4 Figure. Structure of Amphimedon queenslandica DAPK1 ortholog. (A) Structural

560 schematic of $A$. queenslandica DAPK1 (shown to scale). (B) Multiple sequence alignment of the

561 death domain sequences for A. queenslandica DAPK1 and human p75NTR.

562 S5 Table. Large-Scale BLAST queries of PITA and EXT in ecdysozoa and lophotrophozoa.

563 S6 Table. PITAs, EXTs, and Wgn/Grnd homologs spanning bilaterian phyla used for

564 evolutionary modelling.

565 S7 Table. Large-scale BLAST queries of Wgn, Grnd and PITA in protostomian taxa.

566 S8 Figure. Full-length sequence alignments of PITA descendants and human p75NTR. 


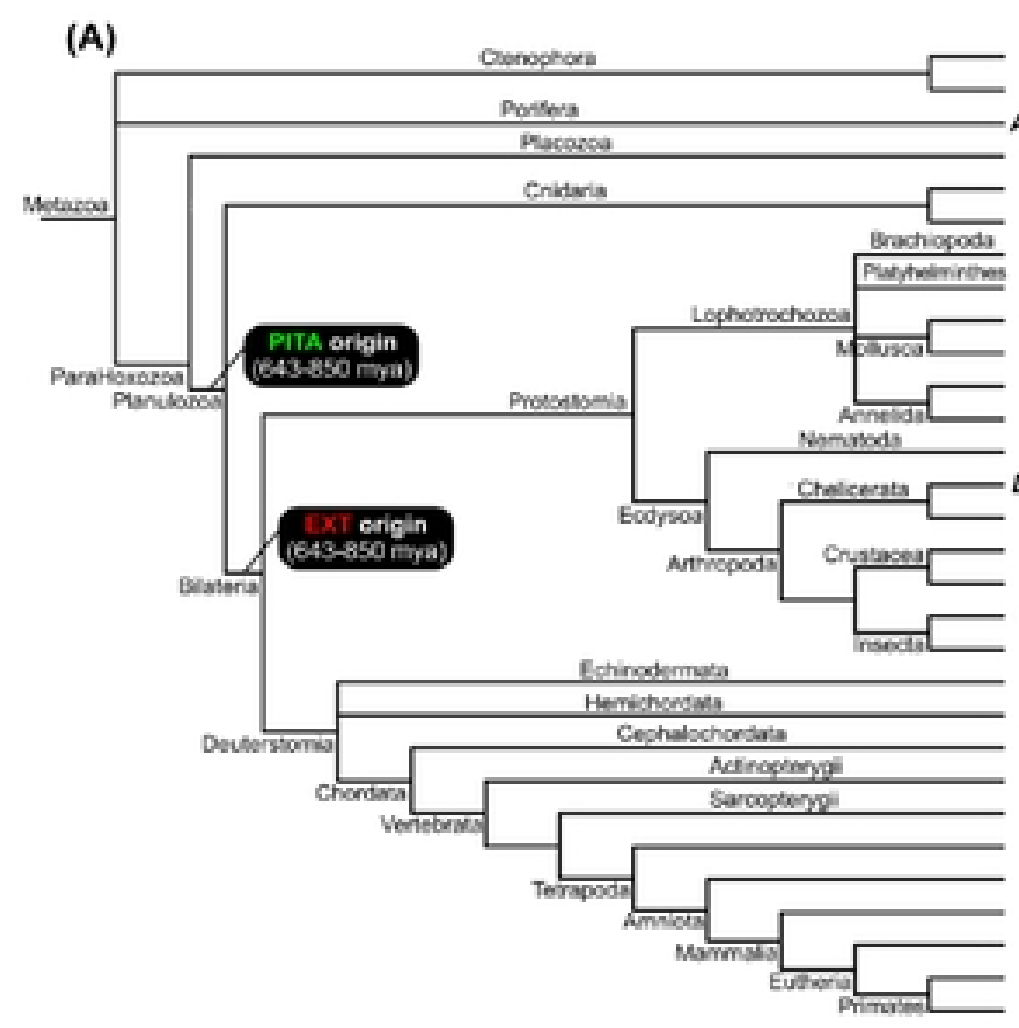

P. bache M. leidy T. adhaerens T. adhaerens N. vectensis L. anatina o. bimacuioides C. giges H. robusta C. eiegans D. magna A. pisum S. purpuratus B. floridae D. rerio
chalumnae $X$. tropicelis G. gaWus O. anatinus M. messculus H. sapiens (B) $\begin{gathered}\text { P75NTR } \\ \text { (it. sapiens) }\end{gathered}$


8 D. pulex

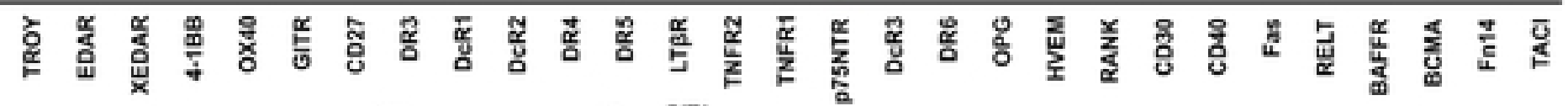
(E) $\underset{\text { (L. anatina) }}{\text { PITA }}$
(F) (CITA

(G) PITA

- Non-p75NTR TNFr(s)

p7SNTR (CRD cluster* TMO-Chopges \&6r DO) pTSNTR (CRD cluster only)

- EDAR, XEDAR \& TROY (CRD cluster) Ortholog structure.

ONNFR-TNFR

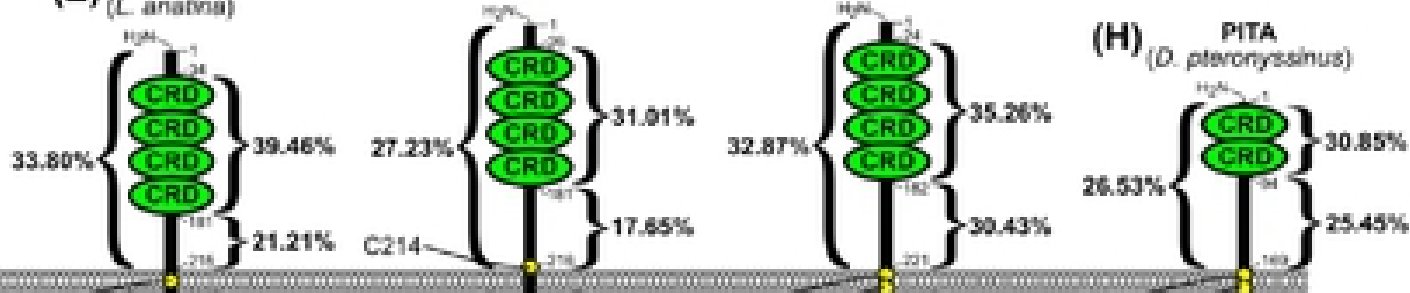

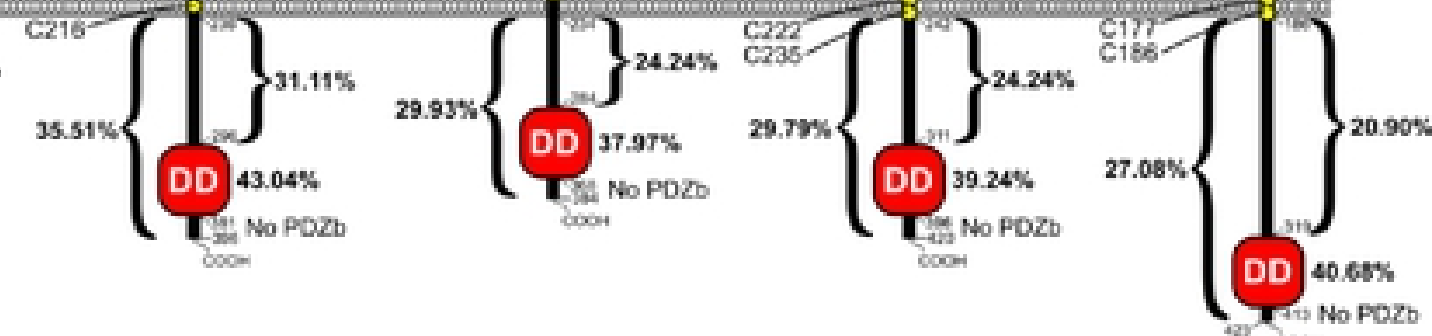

Figure 1 


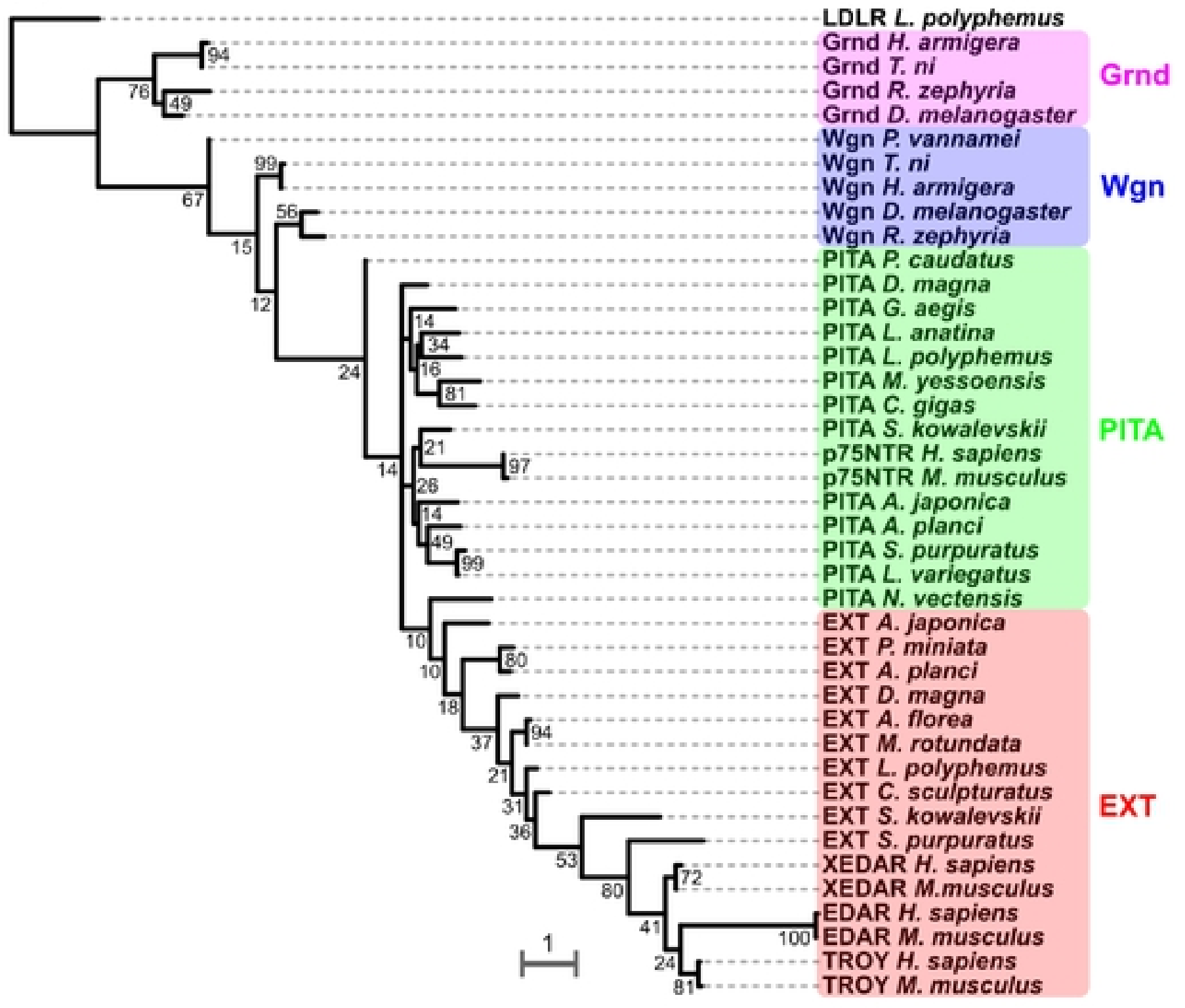

(C) Does species express a DR PITA?
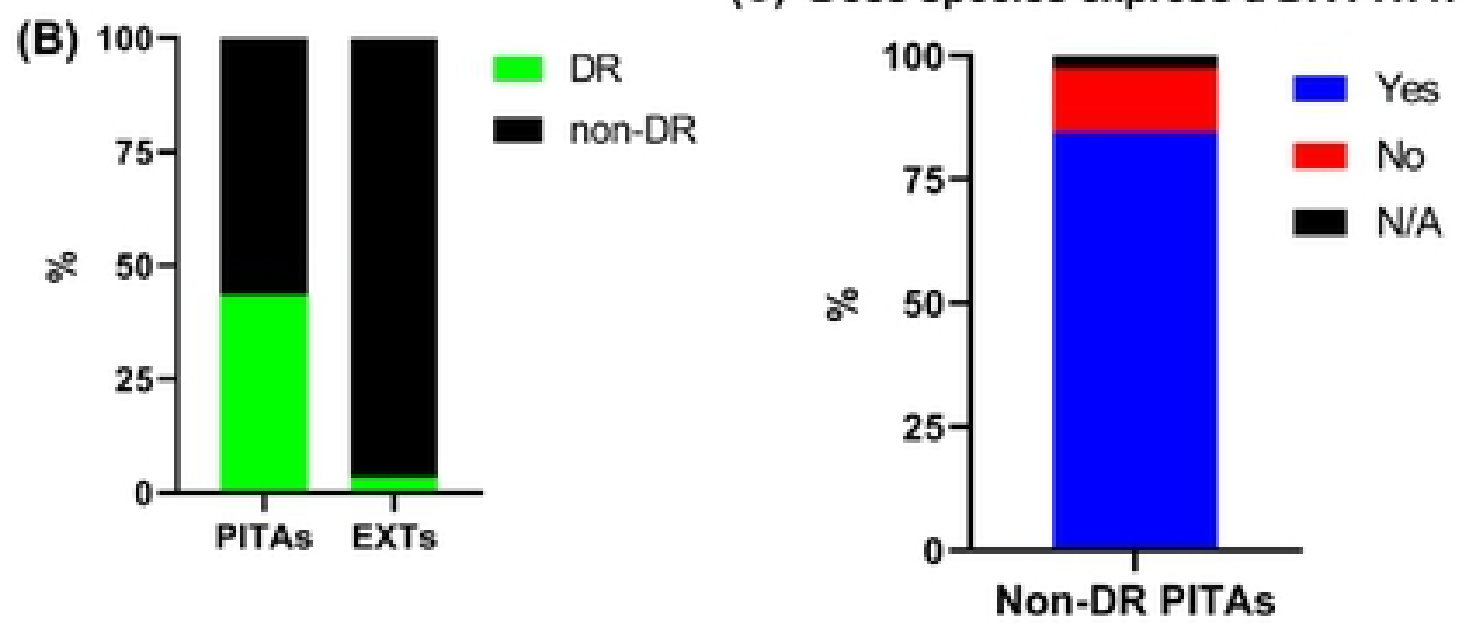

Figure 2 
